\title{
Pharmacokinetics and local safety profile of propranolol eye drops in rabbits
}

Letizia Padrini', Benedetta Isacchi ${ }^{2}$, Anna Rita Bilia ${ }^{2}$, Alessandro Pini ${ }^{3}$, Cecilia Lanzi ${ }^{4}$, Emanuela Masini ${ }^{4}$, Maria Luisa Della Bona ${ }^{5}$, Anna Maria Calvani ${ }^{6}$, Riccardo Ceccantini ${ }^{7}$, Giancarlo la Marca ${ }^{4,5}$ and Luca Filippi ${ }^{1}$

BACKGROUND: Oral propranolol, a nonselective $\beta$-blocker, is able to reduce the progression of retinopathy of prematurity in newborns, but it appeared unsafe. This study aimed to find, in rabbits, a propranolol eye drop concentration able to induce lower plasma but higher retinal concentrations than those obtained after oral administration.

METHODS: Male New Zealand white rabbits were treated with oral propranolol $(0.25 \mathrm{mg} / \mathrm{kg} / 6 \mathrm{~h})$ for $5 \mathrm{~d}$, and propranolol concentrations were measured after 1, 2, 3, and $6 \mathrm{~h}$ in plasma, aqueous humor, vitreous humor, and retina. These concentrations were compared with those obtained after the administration of one drop of $25 \mu \mathrm{l}$ of propranolol $0.1 \%$ prepared in saline, applied every $6 \mathrm{~h}$ to both eyes for $5 \mathrm{~d}$. A Draize eye test and histological analyses were performed to assess eye drop tolerability.

RESULTS: The administration of eye drops produced retinal concentrations similar to, but plasma concentrations significantly lower than, those measured after oral administration. The local tolerability profile was excellent.

CONCLUSION: Propranolol eye drops are able to ensure high retinal and low plasma concentrations of propranolol, and this finding opens the perspective of possible topical treatment with propranolol in newborns with retinopathy of prematurity.

$\mathbf{R}^{\mathrm{s}}$ etinopathy of prematurity (ROP), a vasoproliferative disorder of the retina, is the major cause of blindness and visual impairment in children in both developing and developed countries around the world. Even though the incidence of this disease is closely related to the low birth weight and the low gestational age at birth, preterm infants developing severe ROP in middle- and low-income countries have a wider range of birth weights and gestational ages than what is usually observed in industrialized countries, and this explains why it is so important to counter this disease worldwide (1).

The pathogenesis of ROP consists of two distinct phases. During the first stage of its pathogenesis, the loss of insulin-like growth factor 1 (IGF-1) levels largely produced by the placenta (2) together with exposure to extra-uterine relative hyperoxia suppressing the activity of vascular endothelial growth factor (VEGF) (3) alter normal vascularization of the retina, promoting the vasoconstriction and vaso-obliteration of existing immature vessels (avascular phase of ROP). The second phase of ROP begins at 32-34 wk of postmenstrual age, and is characterized by a hypoxia-induced retinal neovascularization driven by upregulation of VEGF (proliferative phase of ROP) (4). The shift to this second phase has historically been explained by the imbalance between the poorly developed blood vessels and the increasing metabolic demands of the developing retina. This imbalance produces retinal hypoxia, which increases the stability of the inducible $\alpha$ subunit of the transcription factor hypoxia-inducible factor (HIF)-1 and the upregulation of angiogenic growth factors (4).

Recently, the nonselective $\beta_{1} / \beta_{2}$ adrenoreceptor ( $\beta$-AR) antagonist propranolol has been demonstrated to be able to promote the regression of infantile hemangiomas (IHs) (5), and the efficacy of this treatment has been explained on the basis that blockade of $\beta_{2}$-AR inhibits the production of VEGF (6). These findings led us to the hypothesis that inhibition of VEGF through blockade of $\beta_{2}$-AR could also be effective in treating newborns with ROP.

Recent research studies performed in mice with oxygeninduced retinopathy (OIR), a worldwide used model for ROP (7), have demonstrated that norepinephrine accumulation and $\beta$-adrenoreceptor ( $\beta$-AR) activation induce a HIF- $1 \alpha$-driven modulation of VEGF and IGF-1 and participate in mechanisms contributing to the shift from the avascular to the proliferative phase (8-10). Following these observations, two independent prospective pilot trials have demonstrated that oral propranolol administered to preterm newborns suffering from a precocious phase of ROP is effective in counteracting the progression of the disease $(11,12)$, even though not sufficiently safe (11). The ability of propranolol to cross the cornea and reach the periocular tissues in rabbits (13) or the retina of

'Medical Surgical Fetal-Neonatal Department, "A. Meyer" University Children's Hospital, Florence, Italy; ${ }^{2}$ Department of Chemistry, University of Florence, Florence, Italy; ${ }^{3}$ Department of Experimental and Clinical Medicine, University of Florence, Florence, Italy; ${ }^{4}$ Department of Neurosciences, Psychology, Pharmacology and Child Health (NEUROFARBA), University of Florence, Florence, Italy; ${ }^{5}$ Department of Pediatric Neurosciences, "A. Meyer" University Children's Hospital, Florence, Italy; ${ }^{6}$ Department of Pharmacy, "A. Meyer" University Children's Hospital, Florence, Italy; ${ }^{7}$ Department of Pediatric Hematology and Oncology, "A. Meyer" University Children's Hospital, Florence, Italy. Correspondence: Luca Filippi (I.filippi@meyer.it) 
mice with OIR reducing their retinal neovascularization (14) has been recently demonstrated, thus opening the perspective for a possible treatment of ROP with propranolol eye drops.

The present study is aimed at evaluating, in rabbits, the pharmacokinetics of propranolol-saline eye drops, identifying the most appropriate drug concentration to obtain a more favorable retina/plasma ratio of propranolol concentrations compared with oral administration. Subsequently, the local safety profile of eye drops was assessed by performing a Draize eye test in treated animals, and looking for any histological changes in treated tissues.

\section{RESULTS}

\section{Pharmacokinetics of Propranolol After Oral Administration (A Setting)}

In the "A" experimental setting, rabbits were treated with oral propranolol $(1 \mathrm{mg} / \mathrm{kg} / \mathrm{d}$ ) and, after $5 \mathrm{~d}$ of treatment (period of time necessary for reaching steady state), a concentration vs. time profile was performed. The animals at the time of the experiment weighed $3,804 \pm 290 \mathrm{~g}$. Sample measurements are reported in Table 1. In all anatomical analyzed areas, the time of peak concentrations $\left(T_{\max }\right)$ was reached in the first hour after administration, even though the concentrations in aqueous humor were extremely low (Figure 1a).

\section{Ocular Concentration After Topical Administration: The Single- Dose Study (B Setting)}

Propranolol concentrations in plasma, obtained by dried blood spots (DBS), and aqueous humor, after the application of a single eye drop at $0.1,0.5$, and $1 \%$ concentrations, are reported in Table 2. Animals at the time of the experiments weighed $2,055 \pm 290 \mathrm{~g}$. These experiments aimed to analyze the trans-corneal kinetics of propranolol eye drops at different concentrations, and to obtain information useful to choose the concentration for chronic administration (C experimental setting).

After single-drop application, propranolol penetrated corneal layers, reaching the ocular anterior chamber, where maximal concentration $\left(C_{\max }\right)$ was appreciated after $1 \mathrm{~h}$, decreasing subsequently. Drug concentrations at $C_{\max }$ did not vary proportionally to the variation of the solution's concentration, suggesting a nonlinear kinetics. For $0.1 \%$ drops, $6 \mathrm{~h}$ after drop administration, propranolol was undetectable in the anterior chamber. Moreover, propranolol plasma concentration did not

Table 1. Propranolol concentrations at steady state after oral $0.25 \mathrm{mg} / \mathrm{kg} / 6 \mathrm{~h}$ administration

\begin{tabular}{lcccc}
\hline Time & $\begin{array}{c}\text { Plasma } \\
(\mathrm{DBS})(\mu \mathrm{g} / \mathrm{l})\end{array}$ & $\begin{array}{c}\text { Retina } \\
(\mu \mathrm{g} / \mathrm{g})\end{array}$ & $\begin{array}{c}\text { Vitreous } \\
\text { humor }(\mu \mathrm{g} / \mathrm{g})\end{array}$ & $\begin{array}{c}\text { Aqueous } \\
\text { humor }(\mu \mathrm{g} / \mathrm{l})\end{array}$ \\
\hline$T_{0}$ & $2.6 \pm 0.1$ & $21.3 \pm 0.1$ & $1.3 \pm 0.2$ & $0 \pm 0$ \\
$T_{1}$ & $30.1 \pm 8.9$ & $29.2 \pm 5.5$ & $10.2 \pm 4.7$ & $0.4 \pm 0.2$ \\
$T_{2}$ & $17.6 \pm 6.5$ & $26.9 \pm 8.1$ & $2.3 \pm 0.8$ & $0 \pm 0$ \\
$T_{3}$ & $11.1 \pm 6.7$ & $23.1 \pm 7.3$ & $5.2 \pm 2.7$ & $0 \pm 0$ \\
$T_{6}$ & $4.7 \pm 1.6$ & $20.9 \pm 1.2$ & $1.2 \pm 0.4$ & $0 \pm 0$ \\
\hline
\end{tabular}

DBS, dried blood spots. proportionally increase to the growth of the solution's concentration, suggesting a possible limitation to the passage of the drug into circulation after topical administration.

Considering the lower plasma levels obtained after $0.1 \%$ eye drop administration, this concentration was chosen to perform the topical steady state study.

\section{Ocular Concentration After Topical Administration: The Chronic Ocular Pharmacokinetic Study (C Setting)}

Table 3 shows propranolol concentrations in plasma, aqueous humor, vitreous humor, and retina at steady state after 5 $\mathrm{d}$ of topical administration. Animals at the time of the experiment weighed $3,813 \pm 450 \mathrm{~g}$. In this setting, the kinetics were significantly different if compared with oral administration. In fact, propranolol moved quickly from the corneal surface into the ocular anterior chamber and $T_{\max }$ in the aqueous humor was reached at $T_{1}$. Concurrently, drug moved in the posterior direction, and the levels in vitreous humor and in the retina reached $T_{\max }$ at $T_{2}$, and in the plasma, even though the concentrations were extremely low, at $T_{3}$ (Figure 1b). Interestingly, at the end of samplings $\left(T_{6}\right)$, a second spike of propranolol was observed in the retina.

As shown in Figure 2, it was possible to compare the concentration vs. time profiles between oral and ocular administration. While the concentrations at the retinal level were roughly similar (Figure 2d), the concentrations in the plasma were significantly higher after oral administration at $T_{1}, T_{2}$, and $T_{3}(P$ $<0.05$ ) (Figure 2a). When administered topically, propranolol
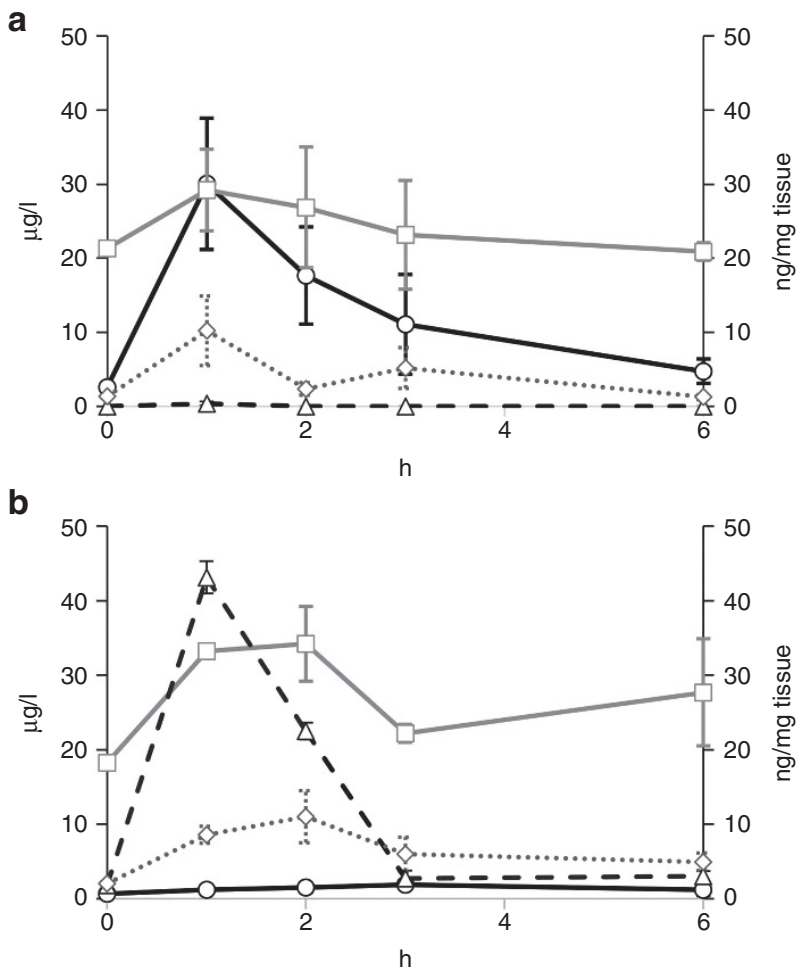

Figure 1. Propranolol concentrations in plasma (solid black line), aqueous humor (dashed black line), vitreous humor (dotted gray line), and retina (solid gray line) after (a) chronic oral or (b) eye drops propranolol administration. 
Table 2. Propranolol concentrations after single ocular drop administration

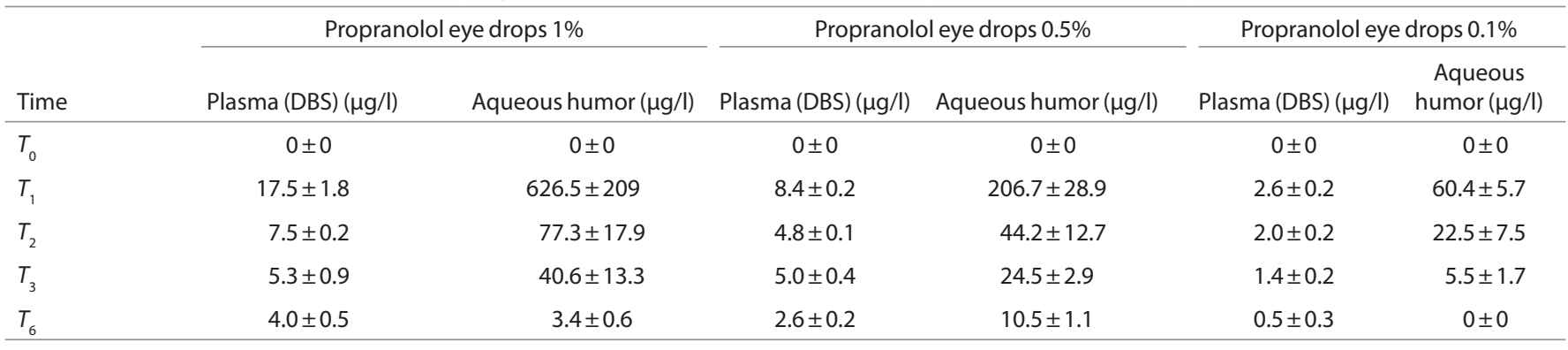

DBS, dried blood spots.

Table 3. Propranolol concentrations at steady state after one $25 \mu \mathrm{l}$ drop/eye/ $6 \mathrm{~h}$ administration

\begin{tabular}{lcccc}
\hline Time & $\begin{array}{c}\text { Plasma } \\
(\mathrm{DBS})(\mu \mathrm{g} / \mathrm{l})\end{array}$ & $\begin{array}{c}\text { Retina } \\
(\mu \mathrm{g} / \mathrm{g})\end{array}$ & $\begin{array}{c}\text { Vitreous } \\
\text { humor }(\mu \mathrm{g} / \mathrm{g})\end{array}$ & $\begin{array}{c}\text { Aqueous } \\
\text { humor }(\mu \mathrm{g} / \mathrm{l})\end{array}$ \\
\hline$T_{0}$ & $0.7 \pm 0.3$ & $18.2 \pm 0.1$ & $2.0 \pm 0.2$ & $1.8 \pm 0.6$ \\
$T_{1}$ & $1.2 \pm 0.5$ & $33.3 \pm 1.0$ & $8.6 \pm 1.1$ & $43.1 \pm 2.1$ \\
$T_{2}$ & $1.5 \pm 0.6$ & $34.2 \pm 5.0$ & $11.0 \pm 3.5$ & $22.5 \pm 1.1$ \\
$T_{3}$ & $1.9 \pm 0.3$ & $22.2 \pm 1.2$ & $6.0 \pm 2.3$ & $2.7 \pm 1.0$ \\
$T_{6}$ & $1.1 \pm 0.7$ & $27.7 \pm 7.2$ & $4.9 \pm 1.1$ & $3.0 \pm 0.7$ \\
\hline
\end{tabular}

DBS, dried blood spots.

reached values significantly higher in aqueous humor at $T_{1}$ and $T_{2}$ (Figure 2b); a similar result was observed in vitreous humor at $T_{2}$ and $T_{6}$ (Figure 2c).

\section{Stability Study}

The chemical stability of propranolol $0.1 \%$ in $\mathrm{NaCl} 0.9 \% \mathrm{w} / \mathrm{v}$ was studied at different temperatures. Propranolol, quantified by LC-MS/MS analysis, maintained a residual percentage of $95.8 \%$ and $97.2 \%$ after $1 \mathrm{wk}$ at $24 \pm 1$ and $37 \pm 1{ }^{\circ} \mathrm{C}$, respectively. Ninety-nine percent of the initial propranolol content was found after $28 \mathrm{~d}$ when the propranolol ophthalmic solutions were kept at $4 \pm 1{ }^{\circ} \mathrm{C}$, recommended as storage conditions.

\section{Local Tolerability: The Draize Eye Test and Histological Analysis}

The Draize test showed no appreciable alterations between control and treated (with propranolol or saline) eyes (null score; Figure 3). Regarding histological analysis, no differences were detected between the corneas (Figure 4a) or the retinas (Figure $4 \mathrm{~b})$ of treated rabbits compared with the untreated ones: treatment with propranolol in fact did not alter the thickness of the inner and outer nuclear layers or the cell number in the ganglion cell layer. Hematoxylin and eosin staining revealed that propranolol administration did not induce significant changes in the corneal and retinal structures (Figure $4 \mathbf{a}-\mathbf{d}$ ). In fact, as revealed by the morphometrical analysis, the number of cells of the ganglion cell layer (GCL) and the thickness of the outer nuclear layer (ONL), the inner nuclear layer (INL) and the inner plexiform layer (IPL) were not significantly modified in the retina of propranolol-administered rabbits compared with the control ones (Figure 4e). Furthermore, propranolol did not alter the thickness or structure of the cornea (Figure $4 \mathrm{f})$.

\section{Microbial Evaluation}

Triplicate spread plates of oral and eye drop preparations showed no bacteria growth (UFC $<1$ ) after $5 \mathrm{~d}$ of incubation at $32.5 \pm 2.5^{\circ} \mathrm{C}$. Inoculation of culture media (for aerobic and anaerobic microorganisms) with both preparations in duplicate showed no growth after $7 \mathrm{~d}$ of incubation at $35.0^{\circ} \mathrm{C}$.

\section{DISCUSSION}

Propranolol, a well-tolerated, nonselective, $\beta$-AR blocker, has been recently reported to induce the involution of IHs (5), the most common tumor of infancy, efficiently and with good tolerance (15). Even though its mechanism of action has not been fully elucidated, recent research demonstrated that the expression of proangiogenic growth factors such as VEGF is downregulated by propranolol in a dose-dependent manner (6).

There is much evidence that the $\beta$-adrenergic system controls angiogenesis through the regulation of proangiogenic factors. Most of the evidence originates from solid tumors and tumor cell lines, in which norepinephrine affects tumor progression by promoting VEGF expression and angiogenesis (16); also in human umbilical vein endothelial cells, and in neonatal rat cardiac myocytes, norepinephrine stimulates angiogenesis by upregulating VEGF production and endothelial proliferation $(17,18)$. $\beta$-ARs are widely expressed on vascular endothelial cells (19), and interesting studies demonstrated that $\beta_{2}$-ARs can regulate VEGF production and neoangiogenesis in response to chronic ischemia (20).

These findings prompted us to verify whether propranolol might be able to reduce the progression of OIR through the downregulation of VEGF. The positive results of these experiments (8-10), confirmed also in mice with choroidal neovascularization (21), prompted us to extend this experimentation to newborns with a precocious stage of ROP as well as (22) a clinical condition driven by high levels of VEGF and frequently associated with IHs (23).

Two clinical trials demonstrated that oral propranolol administered to preterm newborns with a precocious phase of ROP is effective in counteracting the progression of the disease $(11,12)$ but not sufficiently safe. In fact, when propranolol was administered to newborns with very low gestational age and comorbidity (sepsis, surgical interventions requiring general anesthesia) the risk of severe adverse effects (primarily hypotension and bradycardia sometimes unresponsive to catecholamines) was too high, even when dosage was reduced from 
a

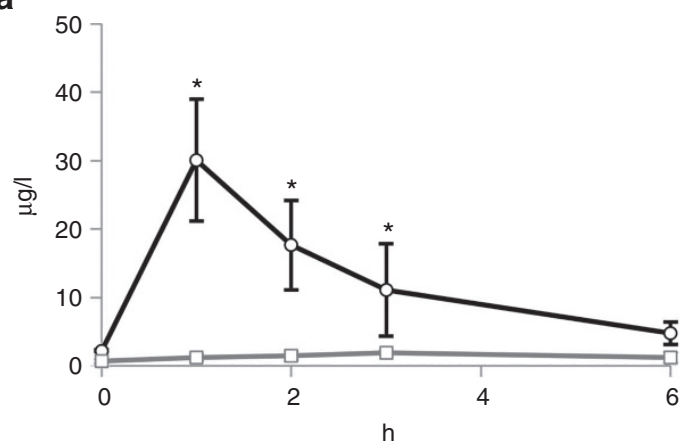

C

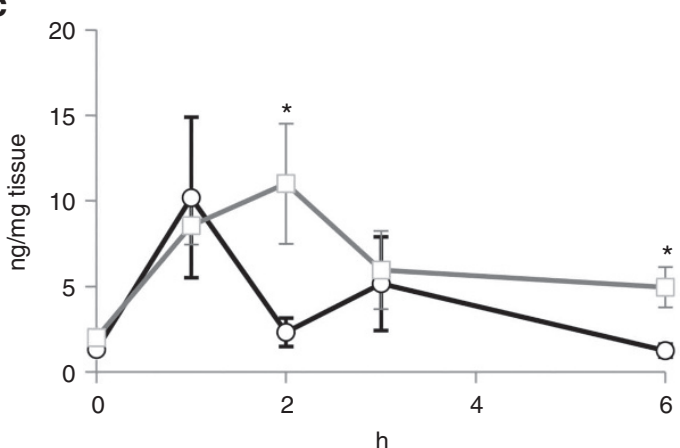

b

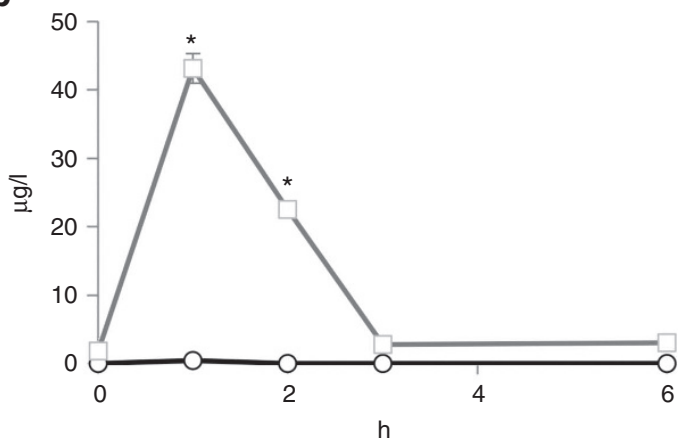

d

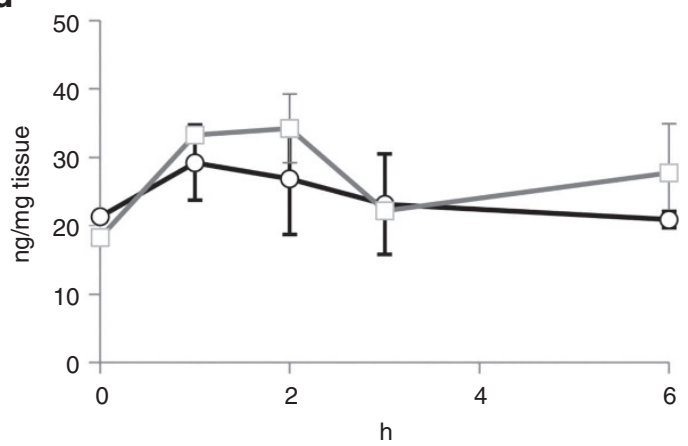

Figure 2. Propranolol concentrations in (a) plasma, (b) aqueous humor, (c) vitreous humor, and (d) retina after chronic oral (solid black line) or eye drops (solid gray line) administration. ${ }^{*} P<0.05$.

the initial dose of $2 \mathrm{mg} / \mathrm{kg} / \mathrm{d}$ to $1 \mathrm{mg} / \mathrm{kg} / \mathrm{d}$ (11). These adverse effects were probably related to propranolol plasma levels and to the long half-life of propranolol in preterm newborns (24). The efficacy of propranolol together with the risk of its systemic administration suggested exploring the perspective of its topical use.

Experiences regarding the use of ophthalmic solution with propranolol are extremely scarce both in humans and in animals. In humans it has been employed at a concentration of $1 \%$ to treat glaucoma; propranolol was found to be effective in lowering intraocular pressure, thus suggesting adequate ocular penetration of the drug, but no data on pharmacokinetics and local tolerability were reported (25). More recently, a study demonstrated the ability of an ophthalmic solution with $1 \%$ propranolol to reach, in rabbits, periocular tissues as well as the anterior ocular segment, with a higher tissue/plasma ratio compared with oral or intravenous administration. Unfortunately, no data were reported about propranolol delivery to the posterior segment of the eye (13). In a recent study, we have demonstrated in mice with OIR that propranolol dissolved in commercially available eye drops ( $2 \%$ concentration) efficiently reaches the retina and promotes the recovery of OIR, suggesting that trans-corneal application of propranolol can lead to adequate penetration of the drug in the posterior segment of the eye (14). However, no specific pharmacokinetic study has been performed at present.

To identify the most appropriate eye drop concentration capable of producing a retina/plasma ratio of propranolol concentrations more favorable than after oral administration, a

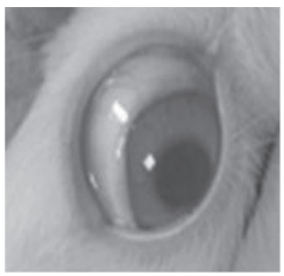

b

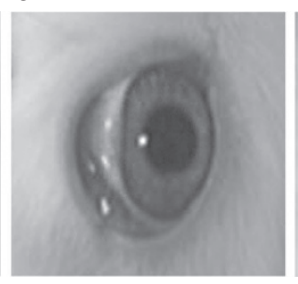

C

Figure 3. Draize test in (a) control rabbits, (b) rabbits treated with saline eye drops, and (c) with propranolol $0.1 \%$ eye drops.

rabbits were initially treated with $1 \mathrm{mg} / \mathrm{kg} / \mathrm{d}$ of oral propranolol, the same dosage used in newborns with lower gestational age enrolled in the clinical trial (11). The results of this first experiment showed high interanimal variability (similar to that reported in neonates) and a $C_{\max }$ very similar to that measured in neonates receiving an equal oral dose, suggesting that this model is appropriate. The minimal concentration $\left(C_{\min }\right)$ observed in rabbits was instead substantially lower than values detected in newborns, and this discrepancy may be explained by the lower metabolism of preterm newborns producing a significantly higher half-life and consequently a higher $C_{\min }(24)$. When propranolol was administered per os, the drug reached the retina easily, but produced very low levels in the vitreous body and is almost absent in aqueous humor.

Usually, ocular topical administration is preferable to systemic administration by delivering high concentrations of drugs directly to the target tissues, minimizing systemic exposure, and avoiding undesirable systemic effects. The ability 


\section{Articles | Propranolol eye drops}

a

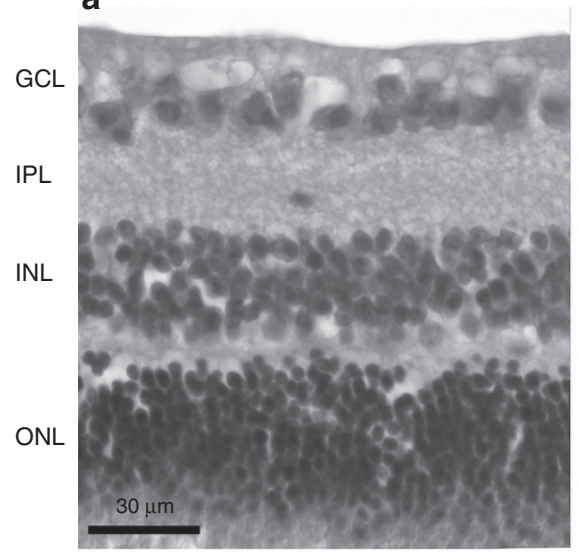

C

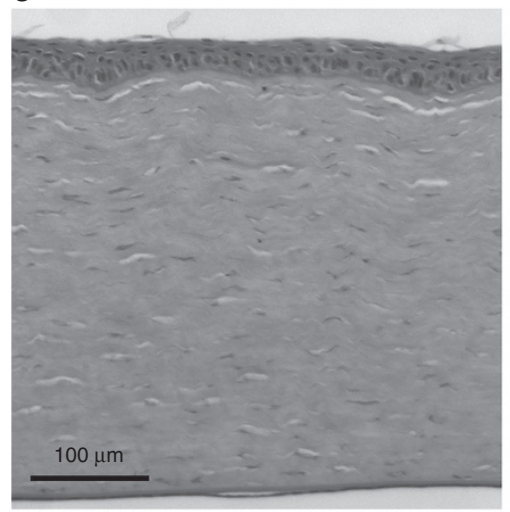

b

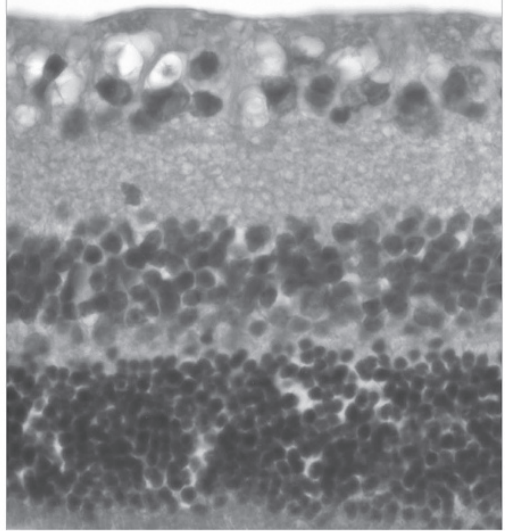

d

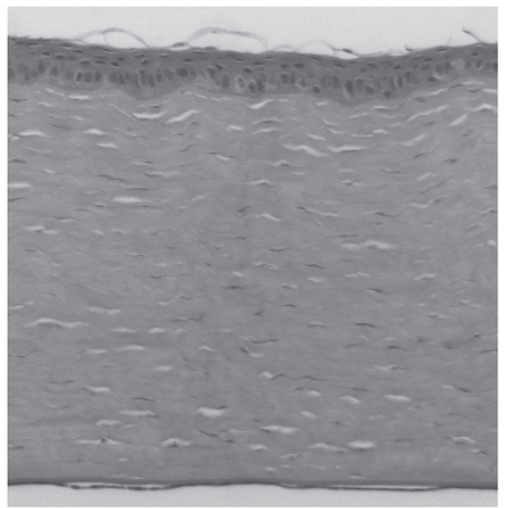

e

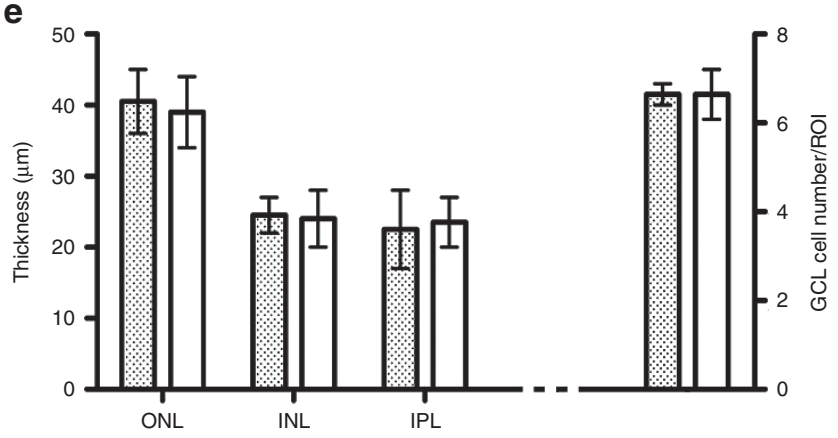

f

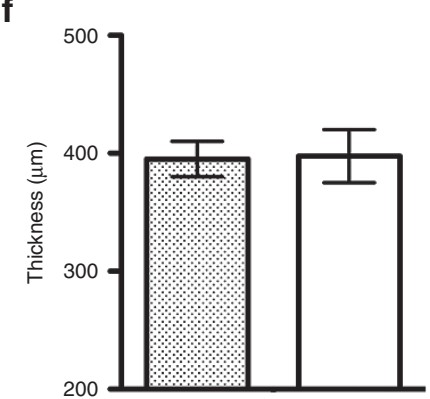

Figure 4. Morphological evaluation of the (a,b) retina and (c,d) cornea of control rabbits (a,c, respectively) and rabbits treated with propranolol $0.1 \%$ eye drops (b,d, respectively). Morphometrical analysis of the (e) retinal layers and (f) corneal thickness of control rabbits (dotted column) and rabbits treated with propranolol $0.1 \%$ eye drops (open column). GCL, ganglion cell layer; INL, inner nuclear layer; IPL, inner plexiform layer; ONL, outer nuclear layer; ROI, region of interest.

of a topical applied drug to pass through the various barriers within the eye is a matter of great interest, especially when the target tissue is located deep in the posterior chamber (i.e., retina). Indeed, it can be hard for a trans-corneal applied drug to reach the retina, because of the presence of many barriers. For this, complex formulation or delivery routes (drug delivery systems, subconjunctival injections) are currently being studied. To date, no studies have been reported on trans-corneal propranolol pharmacokinetics.

In order to plan this pharmacokinetic study, a preliminary test with a single drop at different concentrations was performed. The results demonstrated that saline-based propranolol eye drops adequately penetrate corneal layers and reach the anterior chamber. This is probably due to the high partition coefficient of propranolol; in fact, it is well known that the corneal epithelium does not offer resistance to the penetration of the very lipophilic $\beta$-blockers, such as propranolol (26). After the test with one drop at $1,0.5$, or $0.1 \%$, the concentrations measured did not always change proportionally to the variation of the solution's concentration suggesting a nonlinear kinetics, as previously reported in mice (14). However, the $0.1 \%$ concentration was chosen for chronic administration because the corresponding plasma concentration showed a safer pharmacokinetic profile.

Topical chronic application of $0.1 \%$ propranolol eye drops resulted in a vitreal and retinal concentration of the order of micrograms per gram tissue, indicating that the drug is taken 
up by the tissue from the administration site and conveyed to the posterior segment of the eye. As previously demonstrated, lipophilicity is a key physiochemical property that drives drug distribution within the eye: after intravitreal injection, in fact, lipophilic $\beta$-adrenoreceptor antagonists such as propranolol accumulate in the posterior eye, where they bind to the choroid and the retina, while more hydrophilic $\beta$-adrenoreceptor antagonists such as atenolol preferentially tend to move towards the anterior section of the eye $(27,28)$. Interestingly, the propranolol concentration profile in the retina showed a typical bifid appearance (two spikes at $T_{3}$ and $T_{6}$ ), probably related to propranolol dislodgment from the iris and ciliary bodies and diffusion through the lens and vitreous humor (27).

After oral administration, at steady state, we found that propranolol was rapidly absorbed and distributed to all ocular tissues reaching plasma $C_{\max }$ at $T_{1}$. On the contrary, after topical administration, propranolol reaches $C_{\text {max }}$ progressively later moving from aqueous humor $\left(T_{1}\right)$, to vitreous humor $\left(T_{2}\right)$, to retina $\left(T_{2}\right)$. Plasma $C_{\max }$ is reached after $3 \mathrm{~h}$ from topical administration, probably due to the slow release into the circulation by the cells of the retina and other ocular tissues.

The comparison between the pharmacokinetic results obtained from rabbits employed in settings A (oral chronic administration) and C (ocular chronic administration) represents the most important finding of this study. These data were obtained in rabbits with identical weight, and this comparison showed that chronic topical administration of $0.1 \%$ of propranolol produced retinal concentrations comparable with those obtained after chronic oral $1 \mathrm{mg} / \mathrm{kg} / \mathrm{d}$ administration, but plasma levels about 10-20 times lower, suggesting a better retinal/plasma ratio for ocular drops.

Theoretically, this positive ratio may have been favored by two additional factors. The first is related to the use of eye drops with a volume $(25 \mu \mathrm{l})$ significantly smaller than usual (40-50 $\mu \mathrm{l}$ of standard ocular drops). From the late 1970s, several studies in rabbits and humans demonstrated that reducing ocular drop volume (from 50 to 20 or less), plasma drug concentration and systemic side effects were decreased, while pharmacodynamic effects were conserved $(29,30)$. Authors estimated that precorneal area is able to contain only a small volume of liquid solutions and around $80 \%$ of the volume of a standard drop rapidly reaches the lower conjunctival fornix and passes down the nasolacrimal duct where the drug is systemically absorbed by the highly vascular mucosa (31), thus contributing to systemic side effects. Following these observations, administration of ocular microdrops is currently on study for improving the compliance and safety of ocular preparations in selected patients such as the elderly and neonates $(32,33)$. Therefore, the use of small drops may have contributed to reduce the plasma levels in rabbits. The second factor might be related to the highaffinity binding of lipophilic $\beta$-blockers, such as propranolol, for pigmented tissues (choroid and retinal pigmented epithelium) $(28,34)$. Considering that in this study albino rabbits were used and that in these animals ocular melanin content is very low (28), this factor in this experiment probably did not play a relevant role. However, it is likely that in pigmented humans this property can lead to progressive drug accumulation of propranolol on melanin-containing tissues such as retina. Moreover, pigmented tissues can act as a barrier, limiting the passage of the drug into adjacent tissues: this is, for example, the case of subconjunctival or trans-scleral delivery to the retina of propranolol, which is strongly limited by the choroidal barrier $(28,35)$. In the case of trans-corneal propranolol administration, drug retention in retinal pigmented epithelium can hamper the propranolol passage in plasma and further improve the propranolol retinal/plasma ratio.

Although extensive literature is available concerning the local tolerability profile of various $\beta$-blocker-based eye drops $(36,37)$, to date no studies have analyzed this aspect for propranolol eye drops. In studies in which propranolol-based eye drops were used in humans and mice $(14,25)$, the safety profile was not investigated. With this study we were able to demonstrate that propranolol-based ocular drops at $0.1 \%$ concentration are safe and do not alter ocular structures.

This finding, together with the demonstration of stability of this formulation, is very important because it represents the essential prerequisite for testing these eye drops in neonates with ROP (38).

In conclusion, this study demonstrated that topical administration of $0.1 \%$ propranolol ocular drops in rabbits is safe and produces retinal concentration similar to those found after oral administration, but with plasma concentration significantly lower. Some limitations of this study must be taken into consideration: small numbers of animals used, high interanimal variability, and lack of pharmacodynamic information. Also the choice to use adult rabbits may be questionable, because the pharmacokinetics of propranolol in their eye components might be different from that in newborn rabbit. Although extrapolation of these data to the human condition is difficult considering the differences between human newborns and this animal model, the results of this study facilitate the testing of these eye drops in infants with ROP, as an alternative to less safe oral administration. Similar experimentation might be extended to other proliferative retinal diseases for which oral treatment with propranolol is currently under investigation, such as choroidal hemangioma in Sturge-Weber disease (39), periocular hemangiomas (40), diabetic retinopathy (41), and age-related macular degeneration (42).

\section{METHODS}

\section{Propranolol-Based Preparations}

Oral solution was prepared by diluting propranolol hydrochloride powder purchased from A.C.E.F. (Fiorenzuola d'Arda, Piacenza, Italy),

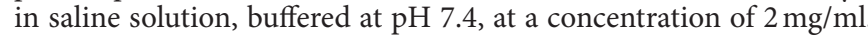
Eye drops were prepared by diluting propranolol hydrochloride powder in saline solution, at a concentration of $0.1,0.5$, and $1 \%$ respectively; these preparations were used for the "single-dose" or "chronic ocular pharmacokinetic studies". Both preparations were prepared sterilely fulfilling microbiological standards requested by European Pharmacopoeia (43). A representative sample of both preparations was tested for sterility with a rapid validated growth test, and blood culture samples were incubated in the BacT Alert 3D (BioMerieux, France) automatized blood culture system. The test system was also validated in the presence of the type of preparation to be examined with respect to specificity and absence of antimicrobial activity. 


\section{Animals}

Experiments were performed on 30 male New Zealand white rabbits, purchased from Harlan Laboratories Italy (Udine, Italy). Animals were kept in a regulated environment $\left(23 \pm 1^{\circ} \mathrm{C}, 50 \pm 5 \%\right.$ humidity), with a 12-h light/dark cycle, with food and water ad libitum. Experiments were performed in compliance with the Italian law on animal care No. 116/1992 and the EEC/609/86. Procedures were approved by the Ethics Committee of Ce.S.A.L, University of Florence. All efforts were made to reduce both animal suffering and the number of animals used.

\section{Pharmacological Treatments}

Three different experimental settings were performed:

A. Oral pharmacokinetic study. Propranolol was administered by using an oropharyngeal tube, at a dose of $0.25 \mathrm{mg} / \mathrm{kg}$ every $6 \mathrm{~h}(1 \mathrm{mg} / \mathrm{kg} / \mathrm{d})$. Propranolol concentrations were measured at the steady state after $5 \mathrm{~d}$ of treatment.

B. Single-dose ocular pharmacokinetic study. To assess optimal drug concentration, different propranolol eye drops were tested. A single $25 \mu$ l propranolol eye drop at a concentration of $0.1,0.5$, and $1 \%$ was applied at each eye just once with a Gilson P50 micropipette, and biological samplings were performed in the following $6 \mathrm{~h}$.

C. Chronic ocular pharmacokinetic study. Propranolol $0.1 \%$ eye drops $(25 \mu \mathrm{l})$ were applied in both eyes, every $6 \mathrm{~h}$, with a Gilson P50 micropipette; biological samplings were performed after $5 \mathrm{~d}$ of treatment, at the steady state.

For settings A and C, biological samples (plasma, aqueous humor, vitreous humor, retina) were collected, killing the animals, immediately before the last eye drop administration $\left(T_{0}\right)$, after $1\left(T_{1}\right), 2\left(T_{2}\right), 3\left(T_{3}\right)$, and $6\left(T_{6}\right) \mathrm{h}$, to obtain a concentration vs. time profile. For setting $\mathrm{B}$, biological samples were limited to blood and aqueous humor, and animals were not sacrificed. For every time point, three animals (six eyes) were used in order to reduce interindividual variability. Animals employed in setting B, after a washout period of at least a week, were subsequently used for settings A or C.

In all experiments, rabbits were anesthetized with a Tiletamine chloridrate/Zolazepam 1:1 solution (Zoletil 100, Virbac, Milan, Italy). Before aqueous humor sampling, local anesthesia was performed by instilling a drop of ossibuprocaine chloridrate (Novesina, Novartis Pharma S.p.a., Origgio, Italy). Blood sampling was performed from the ear's marginal vein, then blood was spotted on a Guthrie card (903, Whatman $\mathrm{GmbH}$, Dassel, Germany) and propranolol was measured from such DBS. Vitreous humor sampling was performed by using insuline-microsyringes (PIC Insumed, Artsana, Grandate, Italy). When sacrifice was requested, rabbits were first anesthetized and then killed with a $200 \mathrm{mg} / \mathrm{kg}$ dose of sodium thiopental (Pentothal sodium, Intervect Productions, Aprilia, Italy). Eyes were enucleated and ocular tissues were separated with a scalpel, operating on ice. Ocular samples (aqueous humor, vitreous humor, retina) were immediately frozen and stored at $-80^{\circ} \mathrm{C}$, until analysis.

\section{Stability Studies}

The stability of propranolol $0.1 \%$ in $\mathrm{NaCl} 0.9 \% \mathrm{w} / \mathrm{v}$ was studied at different temperatures. Propranolol ophthalmic solutions were kept at $+4 \pm 1,+24 \pm 1$, and $+37 \pm 1{ }^{\circ} \mathrm{C}$; at fixed time intervals they were assayed for their chemical stability within $1 \mathrm{wk}$. Moreover, the propranolol solutions stored at $4{ }^{\circ} \mathrm{C}$ were followed over 1 month. Chemical stability was checked by quantification of drug content by HPLC-MS/MS analysis. Each stability test was performed in triplicate.

\section{Draize Eye Test}

To assess the local safety profile, a Draize eye test was performed (44). Six animals were used: for each animal, one eye was instilled with $100 \mu \mathrm{l}$ of $0.1 \%$ propranolol eye drops, once a day, for three consecutive days; the other eye was instilled with the same volume of saline. These eyes were compared with the eyes of nontreated rabbits (control). Ocular response was assessed before treatment $\left(T_{0}\right)$, at $T_{1}, T_{2}$, $T_{3}, T_{6}, T_{24}, T_{48}$, and $24 \mathrm{~h}$ after the last administration $\left(T_{72}\right)$. For each time point, a score was assigned to alterations possibly noted in the iris, conjunctiva, and cornea; considering that corneal lesions impair visual acuity in a greater way, a weighted score system was used (44).

\section{Histological Analysis}

For histological studies, two eyes enrolled in the $\mathrm{C}$ setting were enucleated, washed with saline, fixed in $4 \%$ paraformaldehyde, and paraffin embedded. Histological sections, $7 \mu \mathrm{m}$ thick, were stained with hematoxylin and eosin in a single session to minimize artifactual differences. Photomicrographs of the histological slides were randomly taken with a digital camera connected to a light microscope equipped with $20 \times$ and $40 \times$ objectives. Quantitative assessments of the stained sections were performed by using the free-share ImageJ 1.33 image analysis program (http://rsb.info.nih.gov/ij).

\section{Measurement of Propranolol Concentrations}

Standards. Chemical standard of propranolol hydrochloride (purity $>$ 99\%) was purchased from Sigma-Aldrich (Steinheim, Germany); propranolol-d7 (97.8\% D purity) was from CDN Isotopes (Quebec, Canada). Stock solutions, prepared in methanol and collected at -20 ${ }^{\circ} \mathrm{C}$, were 16.2 and $11.3 \mathrm{mmol} / \mathrm{l}$, respectively. Successive dilutions were made using HPLC-grade water. HPLC-grade methanol, acetonitrile, and water were purchased from Panreac (Barcelona, Spain).

Blood samples. Plasma propranolol concentrations were measured on DBS by the liquid-chromatography tandem-mass spectrometry (LC$\mathrm{MS} / \mathrm{MS}$ ) test, as previously reported (45).

Aqueous humor samples. A $5 \mu \mathrm{l}$ volume of each sample was added to $200 \mu \mathrm{lmethanol} /$ water $95: 5(\mathrm{v} / \mathrm{v})+0.1 \%$ formic acid solution containing $1 \mu \mathrm{g} / \mathrm{l}$ of propranolol-d7 and shaken for $10 \mathrm{~s}$ by vortex. The solution was analyzed by LC-MS/MS.

Vitreous humor samples. A $100 \mu \mathrm{l}$ volume of vitreous humor was added to $200 \mu \mathrm{l}$ water $+1.5 \%$ ammonia solution and $10 \mu \mathrm{l}$ of propranolol-d7 $(200 \mu \mathrm{g} / \mathrm{l})$, then shaken for $10 \mathrm{~s}$ by vortex. A double liquidliquid extraction with $500 \mu \mathrm{l}$ of hexane was performed; supernatant was transferred into a new vial, dried under nitrogen flow, and reconstituted with $200 \mu \mathrm{l}$ methanol/water 95/5 (v/v).

Retina samples. To the retina sample, $600 \mu \mathrm{l}$ of water and $10 \mu \mathrm{l}$ of propranolol-d7 $(10 \mu \mathrm{mol} / \mathrm{l})$ were added. Sample homogenization was performed using a mechanical homogenizer (POLYTRON PT 2100 Homogenizers, Kinematica), followed by ultrasonication in the ice bath for $5 \mathrm{~min}$. A $200 \mu \mathrm{l}$ volume of the homogenate was purified by a silica minicolumn (NucleoSpin TriPrep, Macherey-Nagel, Germany) and then centrifuged at $10,000 \mathrm{~g}$ in a microfuge for $10 \mathrm{~min}$. The minicolumn was washed with $300 \mu$ l of ethyl acetate, two times, and $400 \mu \mathrm{l}$ of hexane. This organic phase was removed and the sample was eluted with $200 \mu \mathrm{l}$ of methanol/water $1 / 1(\mathrm{v} / \mathrm{v})$.

Mass spectrometry. The LC-MS/MS method for the quantification of propranolol has been recently reported $(24,45)$. Propranolol concentrations in DBS and aqueous humor are expressed as $\mu \mathrm{g} / \mathrm{l}$, and in retina and vitreous humor samples as $\mu \mathrm{g} / \mathrm{g}$.

\section{Statistical Analysis}

Data were reported as mean \pm SEM and originated from three animals (six eyes) for each experimental condition. Unless otherwise stated, statistical significance was determined by one-way ANOVA with the Newman-Keuls multiple comparison test, two-way ANOVA, or the Student's $t$-test. A $P$ value $<0.05$ was considered statistically significant. Calculations were performed using GraphPad Prism software (GraphPad, San Diego, CA).

\section{STATEMENT OF FINANCIAL SUPPORT}

This study was supported by Ente Cassa di Risparmio di Firenze, Florence, Italy.

Disclosure: The authors confirm that there is no potential, perceived, or real conflict of interest.

\section{REFERENCES}

1. Gilbert C. Retinopathy of prematurity: a global perspective of the epidemics, population of babies at risk and implications for control. Early Hum Dev 2008;84:77-82.

2. Hellström A, Engström E, Hård AL, et al. Postnatal serum insulin-like growth factor I deficiency is associated with retinopathy of prematurity and other complications of premature birth. Pediatrics 2003;112:1016-20. 
3. Penn JS, Madan A, Caldwell RB, Bartoli M, Caldwell RW, Hartnett ME. Vascular endothelial growth factor in eye disease. Prog Retin Eye Res 2008;27:331-71.

4. Pierce EA, Foley ED, Smith LE. Regulation of vascular endothelial growth factor by oxygen in a model of retinopathy of prematurity. Arch Ophthalmol 1996;114:1219-28.

5. Léauté-Labrèze C, Dumas de la Roque E, Hubiche T, Boralevi F, Thambo JB, Taïeb A. Propranolol for severe hemangiomas of infancy. N Engl J Med 2008;358:2649-51.

6. Ji Y, Li K, Xiao X, Zheng S, Xu T, Chen S. Effects of propranolol on the proliferation and apoptosis of hemangioma-derived endothelial cells. J Pediatr Surg 2012;47:2216-23.

7. Chen J, Smith LE. Retinopathy of prematurity. Angiogenesis 2007;10:13340.

8. Ristori C, Filippi L, Dal Monte M, et al. Role of the adrenergic system in a mouse model of oxygen-induced retinopathy: antiangiogenic effects of beta-adrenoreceptor blockade. Invest Ophthalmol Vis Sci 2011;52:155-70.

9. Martini $\mathrm{D}$, Monte $\mathrm{MD}$, Ristori $\mathrm{C}$, et al. Antiangiogenic effects of $\beta 2$-adrenergic receptor blockade in a mouse model of oxygen-induced retinopathy. J Neurochem 2011;119:1317-29.

10. Dal Monte M, Martini D, Latina V, Pavan B, Filippi L, Bagnoli P. Betaadrenoreceptor agonism influences retinal responses to hypoxia in a model of retinopathy of prematurity. Invest Ophthalmol Vis Sci 2012;53:2181-92.

11. Filippi L, Cavallaro G, Bagnoli P, et al. Oral propranolol for retinopathy of prematurity: risks, safety concerns, and perspectives. J Pediatr 2013;163:1570-7.

12. Makhoul IR, Peleg O, Miller B, et al. Oral propranolol versus placebo for retinopathy of prematurity: a pilot, randomised, double-blind prospective study. Arch Dis Child 2013;98:565-7.

13. Hao J, Yang MB, Liu H, Li SK. Distribution of propranolol in periocular tissues: a comparison of topical and systemic administration. J Ocul Pharmacol Ther 2011;27:453-9.

14. Dal Monte M, Casini G, la Marca G, Isacchi B, Filippi L, Bagnoli P. Eye drop propranolol administration promotes the recovery of oxygen-induced retinopathy in mice. Exp Eye Res 2013;111:27-35.

15. Schiestl C, Neuhaus K, Zoller S, et al. Efficacy and safety of propranolol as first-line treatment for infantile hemangiomas. Eur J Pediatr 2011;170:493501.

16. Park SY, Kang JH, Jeong KJ, et al. Norepinephrine induces VEGF expression and angiogenesis by a hypoxia-inducible factor- $1 \alpha$ protein-dependent mechanism. Int J Cancer 2011;128:2306-16.

17. Seya Y, Fukuda T, Isobe K, Kawakami Y, Takekoshi K. Effect of norepinephrine on RhoA, MAP kinase, proliferation and VEGF expression in human umbilical vein endothelial cells. Eur J Pharmacol 2006;553:54-60.

18. Weil J, Benndorf R, Fredersdorf S, Griese DP, Eschenhagen T. Norepinephrine upregulates vascular endothelial growth factor in rat cardiac myocytes by a paracrine mechanism. Angiogenesis 2003;6:303-9.

19. Guimarães S, Moura D. Vascular adrenoceptors: an update. Pharmacol Rev 2001;53:319-56.

20. Iaccarino G, Ciccarelli M, Sorriento D, et al. Ischemic neoangiogenesis enhanced by beta2-adrenergic receptor overexpression: a novel role for the endothelial adrenergic system. Circ Res 2005;97:1182-9.

21. Lavine JA, Sang Y, Wang S, Ip MS, Sheibani N. Attenuation of choroidal neovascularization by $\beta(2)$-adrenoreceptor antagonism. JAMA Ophthalmol 2013;131:376-82.

22. Filippi L, Cavallaro G, Fiorini P, et al. Study protocol: safety and efficacy of propranolol in newborns with Retinopathy of Prematurity (PROP-ROP): ISRCTN18523491. BMC Pediatr 2010;10:83.

23. Praveen V, Vidavalur R, Rosenkrantz TS, Hussain N. Infantile hemangiomas and retinopathy of prematurity: possible association. Pediatrics 2009;123:e484-9.
24. Filippi L, Cavallaro G, Fiorini P, et al. Propranolol concentrations after oral administration in term and preterm neonates. J Matern Fetal Neonatal Med 2013;26:833-40.

25. Vale J, Gibbs AC, Phillips CI. Topical propranolol and ocular tension in the human. Br J Ophthalmol 1972;56:770-5.

26. Shih RL, Lee VH. Rate limiting barrier to the penetration of ocular hypotensive beta blockers across the corneal epithelium in the pigmented rabbit. J Ocul Pharmacol 1990;6:329-36.

27. Mains J, Tan LE, Wilson C, Urquhart A. A pharmacokinetic study of a combination of beta adrenoreceptor antagonists - in the isolated perfused ovine eye. Eur J Pharm Biopharm 2012;80:393-401.

28. Kadam RS, Kompella UB. Influence of lipophilicity on drug partitioning into sclera, choroid-retinal pigment epithelium, retina, trabecular meshwork, and optic nerve. J Pharmacol Exp Ther 2010;332:1107-20.

29. Patton TF, Francoeur M. Ocular bioavailability and systemic loss of topically applied ophthalmic drugs. Am J Ophthalmol 1978;85:225-9.

30. File RR, Patton TF. Topically applied pilocarpine. Human pupillary response as a function of drop size. Arch Ophthalmol 1980;98:112-5.

31. Shell JW. Pharmacokinetics of topically applied ophthalmic drugs. Surv Ophthalmol 1982;26:207-18.

32. Elibol O, Alçelik T, Yüksel N, Caglar Y. The influence of drop size of cyclopentolate, phenylephrine and tropicamide on pupil dilatation and systemic side effects in infants. Acta Ophthalmol Scand 1997;75:178-80.

33. Kumar S, Karki R, Meena M, Prakash T, Rajeswari T, Goli D. Reduction in drop size of ophthalmic topical drop preparations and the impact of treatment. J Adv Pharm Technol Res 2011;2:192-4.

34. Pitkänen L, Ranta VP, Moilanen H, Urtti A. Binding of betaxolol, metoprolol and oligonucleotides to synthetic and bovine ocular melanin, and prediction of drug binding to melanin in human choroid-retinal pigment epithelium. Pharm Res 2007;24:2063-70.

35. Kadam RS, Cheruvu NP, Edelhauser HF, Kompella UB. Sclera-choroidRPE transport of eight $\beta$-blockers in human, bovine, porcine, rabbit, and rat models. Invest Ophthalmol Vis Sci 2011;52:5387-99.

36. Zimmerman TJ, Kass MA, Yablonski ME, Becker B. Timolol maleate: efficacy and safety. Arch Ophthalmol 1979;97:656-8.

37. Long DA, Johns GE, Mullen RS, et al. Levobunolol and betaxolol. A double-masked controlled comparison of efficacy and safety in patients with elevated intraocular pressure. Ophthalmology 1988;95:735-41.

38. The Italian Medicines Agency. Safety and Efficacy of Propranolol Eye Drops in Treating Retinopathy of Premature (DROP-PROP), 2013. (http:// clinicaltrials.gov/show/NCT02014454).

39. Arevalo JF, Arias JD, Serrano MA. Oral propranolol for exudative retinal detachment in diffuse choroidal hemangioma. Arch Ophthalmol 2011;129:1373-5.

40. Missoi TG, Lueder GT, Gilbertson K, Bayliss SJ. Oral propranolol for treatment of periocular infantile hemangiomas. Arch Ophthalmol 2011;129:899-903.

41. University of Wisconsin, Madison. Propranolol for Diabetic Retinopathy. 2012. (http://clinicaltrials.gov/show/NCT01535495).

42. Montero JA, Ruiz-Moreno JM, Sanchis-Merino E, Perez-Martin S. Systemic beta-blockers may reduce the need for repeated intravitreal injections in patients with wet age-related macular degeneration treated by bevacizumab. Retina 2013;33:508-12.

43. Council of Europe. Microbiological examination of non-sterile products (total viable aerobic count). In: Council of Europe, eds. European Pharmacopoeia 5th edn. Strasbourg: Council of Europe,200520612:154-6.

44. Wilhelmus KR. The Draize eye test. Surv Ophthalmol 2001;45:493-515.

45. Della Bona ML, Malvagia S, Villanelli F, et al. A rapid liquid chromatography tandem mass spectrometry-based method for measuring propranolol on dried blood spots. J Pharm Biomed Anal 2013;78-79:34-8. 